\title{
Coulisses
}

Revue de théâtre

12 | Printemps 1995

Varia

\section{Claude Nicolas Ledoux et le Théâtre de Besançon}

\section{Patrick Melior}

\section{OpenEdition}

Journals

Édition électronique

URL : http://journals.openedition.org/coulisses/3831

DOI : 10.4000/coulisses.3831

ISSN : 2546-9460

\section{Éditeur}

Presses universitaires de Franche-Comté

\section{Édition imprimée}

Date de publication : 1 mai 1995

Pagination : 73-77

ISSN : 1150-594X

\section{Référence électronique}

Patrick Melior, «Claude Nicolas Ledoux et le Théâtre de Besançon », Coulisses [En ligne], 12 |

Printemps 1995, mis en ligne le 15 mars 2019, consulté le 21 octobre 2019. URL : http:// journals.openedition.org/coulisses/3831 ; DOI : 10.4000/coulisses.3831

Ce document a été généré automatiquement le 21 octobre 2019

Coulisses 


\section{Claude Nicolas Ledoux ${ }^{1}$ et le Théâtre de Besançon}

\section{Patrick Melior}

«Ces hommes tout à leur siècle, ne jouiront jamais du consolant espoir de l'avenir. " C. Nicolas Ledoux.

Le Théâtre de Besançon (1775-1784)

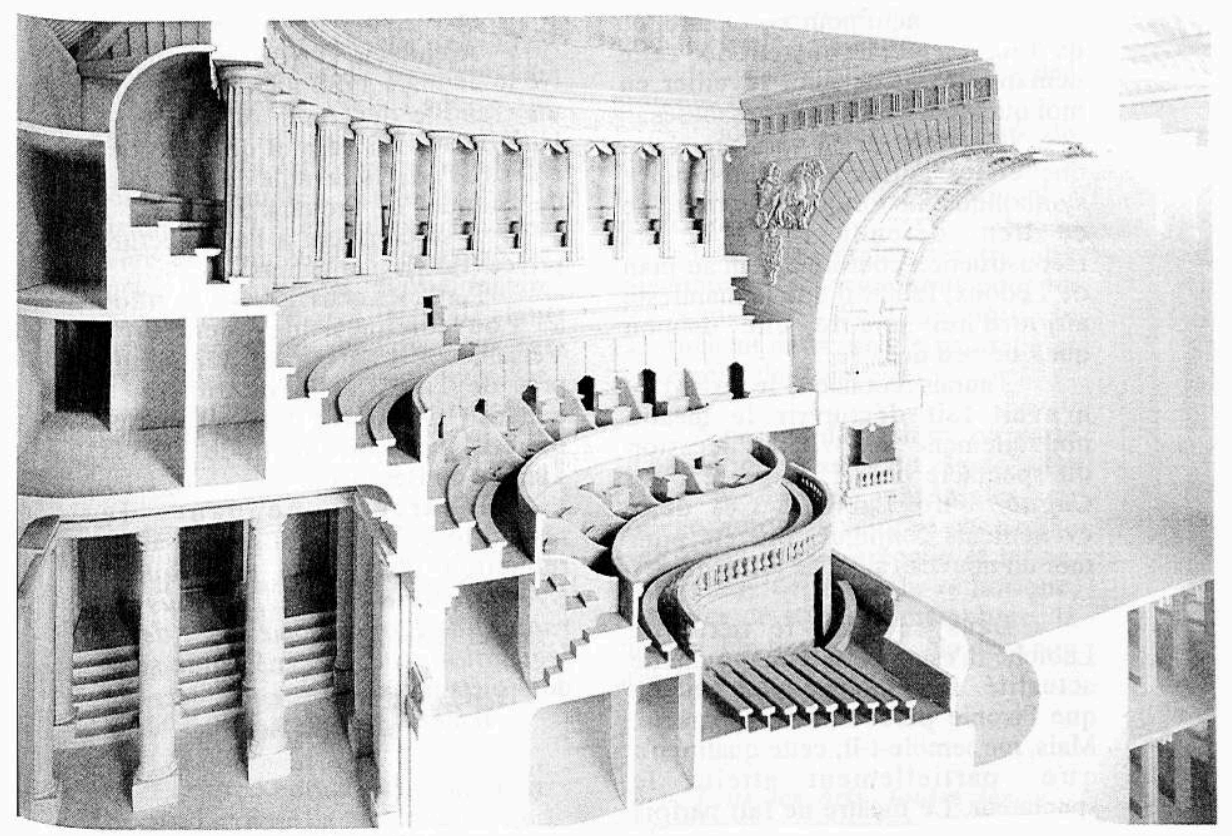

Photographie Georgs Fessy - (c) Fondation Claude Nicolas Ledoux

Il y a quelque temps, Lucile Garbagnati me demandait, pour la revue Coulisses un article sur Claude Nicolas Ledoux et le théâtre de Besançon. Ceux qui connaissent mon 
attachement pour ce théâtre, ce qu'il représente, devineront que cette demande avait de quoi réveiller en moi quelques sentiments désabusés.

Après avoir en vain alerté les uns et les autres sur l'importance symbolique, historique, théâtrale, de ce lieu, défendu l'idée de sa reconstruction conformément au plan de Ledoux, fallait-il que je manifeste aujourd'hui encore une opinion quelque peu décalée?

J'aurais renoncé si le hasard ne m'avait fait découvrir le théâtre nouvellement « rénové » à l'occasion du spectacle de J.-L. Lagarce : La Cagnotte de Labiche. Ces deux événements conjugués ont été pour moi un nouveau sujet de réflexion.

Dans ce lieu, le texte de Labiche m'est apparu d'une étonnante actualité. La violence plus encore que l'ironie parcourait le spectacle. Mais, me semble-t-il, cette qualité n'a que partiellement atteint le spectateur. Le théâtre ne fait parfois qu'effleurer les consciences. Le public sort comme il entre, indemne.

Pourtant les métaphores étaient là :

La Cagnotte, les coups de pioche, le morceau de corniche, La Ferté-sous-Jouarre. Tous les « menus plaisirs » d'un théâtre « embelli ».

Mais revenons à Claude Nicolas Ledoux. Comprendre son théâtre exige que l'on s'intéresse à l'homme et son temps.

"On peut être vertueux ou vicieux comme le caillou rude ou poli, par le

frottement de ce qui nous entoure."

C. Nicolas Ledoux

\section{Qui est Claude Nicolas Ledoux?}

4 Architecte et graveur français. Né le 27 mars 1736 à Dormans dans une famille de condition modeste. Une bourse lui permet de faire des études à Paris. Il travaille dans l'atelier de Blondel et Trouart. De 1762 à 1774, il se consacre à l'architecture privée (Hôtels particuliers).

Parmi ses œuvres : un pavillon à Louveciennes, des écuries à Versailles, le Château de Bénouville près de Caen, les Salines d'Arc-et-Senans (1774), en 1785 les barrières de Paris. Il conçoit à partir de 1775 le Théâtre de Besançon.

Incarcéré pendant la révolution, il se consacre à la rédaction d'un texte théorique : L'Architecture considérée sous le rapport de l'art, des mœurs et de la législation, accompagné d'une série de projets utopiques.

Il meurt à Paris en 1806.

« Ledoux a pressenti, sous le règne de Louis XV, l'avènement d'une ère sociale nouvelle.»

Hélène Leclerc

\section{Claude Nicolas Ledoux et l'histoire moderne}

Bien avant la révolution et jusqu'à nos jours, l'œuvre architecturale de Claude Nicolas Ledoux aura été le champs d'affrontements violents. Elle provoquait des réactions polémiques et esthétiques.

De son vivant Ledoux s'était fait la réputation d'un inventeur brillant mais fantasque. 
Victor Hugo vers 1830 lui reprochait d'avoir réduit son art à une " vulgaire géométrie ». Il fallut attendre les années 1920 pour mettre fin à ces critiques. Ledoux apparut alors comme le précurseur de le Corbusier, (cf. : Emil Kaufmann) héros du modernisme. Dans les années 1960 Ledoux fut associé au socialisme utopique. Aujourd'hui il est l'objet d'imitations post-modernistes. Ledoux fut-il visionnaire ? Bien qu'appartenant à « l'ère des lumières ", Ledoux ne fut jamais politiquement révolutionnaire mais son œuvre architecturale, tant par ses aspirations utopiques que par ses remises en causes formelles, « anticipait les profondes mutations de la société moderne ».

"A quoi servent les connaissances, si elles ne rendent pas meilleurs ?»

C. Nicolas Ledoux

\section{Ledoux, graveur, architecte et philosophe}

Pour Ledoux, comme pour Diderot, la vérité en architecture c'est révéler, exprimer « le caractère » propre à chaque édifice.

Son ambition fut de surmonter la division, prétendument irrémédiable, entre la destination d'un bâtiment et sa représentation.

Le bâtiment doit donc à la fois remplir une fonction qui lui est propre et en être le signe transparent.

Ledoux fut apprenti graveur. Ses connaissances architecturales lui venaient de la gravure.

Sa vision globale de l'architecture il la doit sans doute à l'exercice de ce mode unificateur de la représentation.

Ainsi Ledoux s'est constitué un lexique architectural non pas à partir de modèles historiques existants mais de leurs représentations.

Ce goût pour le graphisme, le dessin, les "réalités virtuelles ", a fait de lui un architecte sensible aux jeux de l'espace, aux systèmes formels, aux structures, à la géométrie.

Cette disposition d'esprit a des prolongements philosophiques.

Ledoux avait beaucoup de sympathie pour les idéaux esthétiques et éthiques des encyclopédistes.

Les fêtes rurales, les travaux saisonniers, l'artisanat sont pour lui des souvenirs d'enfance qui le rapprochent de la morale naturelle et universelle de Rousseau.

7 A propos de Ledoux on parle "d'utopie de la salle de classe ». En effet, ses maîtres jansénistes, au collège, auront développé chez lui le goût de la poésie grecque et latine, mais aussi celui de la rhétorique, l'exercice de style, la métaphore, la citation. On trouve tout cela dans son architecture.

«Tous les arts, toutes les conditions trouvent des demeures qui leur sont propres."

C. Nicolas Ledoux

\section{Le théâtre à l'époque de Ledoux}

A partir du XVIII ${ }^{\text {ème }}$ siècle, l'autonomie fondamentale entre une vision populaire et une conception aristocratique du théâtre est mise en cause.

Un véritable débat sur la théâtralité va passionner les philosophes (Diderot, Rousseau) 
et les architectes (Soufflot, De Wailly, Ledoux).

Il n'est plus question alors de diviser l'activité théâtrale entre d'une part "des représentations privées, données dans les appartements d'un hôtel aristocrate, et d'autre part des spectacles populaires pour la place du marché, la foire. »

La théâtralité dorénavant va "s'incarner dans un monument publique susceptible de confirmer la place du spectacle dans le cadre des loisirs de la bourgeoisie provinciale. »

Le théâtre va devenir un domaine spécialisé et professionnalisé ou un public discipliné apprendra à goûter « les valeurs esthétiques de l'art dramatique ».

Cette théâtralité ou vision du théâtre va donner lieu à une forme spécifique de bâtiment : "Le Théâtre ", cela aux dépens à la fois des traditions aristocratiques mais aussi populaires.

Ce débat sur la réforme du théatre public, sur l'esthétique de la «mise en scène », sur les conséquences sociales et morales de la représentation, a un modèle : l'antiquité.

«Il s'agit de renouer avec l'antiquité en rétablissant le théâtre dans sa fonction pédagogique et sociale. Cela implique une politique culturelle nationale avec des écoles de comédiens, et une nouvelle architecture des salles. "

J.C Bonnet

«Le langage sévère, hermétique dans sa simplicité, d'un Ledoux au théâtre de Besançon, porte en lui toutes les virtualités futures. "

Hélène Leclerc

\section{Les innovations de Ledoux au théâtre de Besançon}

La vision de Ledoux concernant le théâtre était moins pittoresque que sociale : à ses yeux, le théâtre devait prendre sa place au sein des institutions moralisatrices de la société, devait avant tout devenir lieu « d'instruction publique ».

Comme lui, les architectes Soufflot et De Wailly vont défendre, au nom du caractère naturel, le plan antique donc l'amphithéâtre romain.

Obéissant à ces "lois naturelles " qui privilégient le cercle ("assis avec les dieux ») Ledoux s'oppose à la demande sociale de loges privées, «les vastes volières" hiérarchiquement distribuées selon des critères de rang et d'apparat.

L'aristocratie parlera à ce propos de « crime contre la hiérarchie ». Le projet de Ledoux à Besançon sera donc conforme à ce qu'il nomme « le système progressif » :

\section{Bouleversement de l'ordre traditionnel des sièges}

- Le parterre (« Le parc moutonnier ») est transféré au « paradis ou poulailler ».

- Le «nouveau parterre », rebaptisé parquet, est un amphithéâtre. Le public y est assis («on jugera plus sereinement les auteurs »).

- Les sièges d'honneur, qui étaient sur la scène elle-même, sont supprimés («On préfère la pièce au public »).

- Pas de loges fermées (répondant ainsi aux critiques de Rousseau dans sa lettre à d'Alembert, contre la licence qui régnait dans les théâtres).

- La transparence viendra donc renforcer la morale. 


\section{Les qualités acoustiques}

- La géométrie semi-sphérique du théâtre devait répercuter le son à travers la salle, imitant ainsi «l'harmonie universelle du monde».

- La disposition de l'orchestre: une fosse («le fossé magique» repris par R. Wagner à Bayreuth) située entre le "parquet" et la scène devait permettre de supprimer tout obstacle visuel entre le public et la scène (occultation de l'orchestre).

- De même l'équilibre des masses sonores entre la voix et l'orchestre sera mieux maîtrisé. (le son se réfléchissait contre une chicane en bois incurvée)

\section{La place des illusions} reprises par Servandoni en France, dans la plantation du décor : l'usage des praticables qui viennent rompre la monotonie des lignes fuyant vers un même point. Mais son souci est encore différent du leur, sa conception n'est pas simplement celle d'un décorateur, mais d'un véritable «metteur en scène » (au sens moderne du terme) soucieux du rapport plastique entre l'acteur et les objets qui l'entourent.

\section{Le « corps lisse »}

11 Ledoux souligne l'importance de l'espace de transition entre le réel et le fictif, la société et ses illusions.

Le cadre de scène doit donc être ce « corps lisse », sans obstacle, cet « œil » ou « cadre naturel », cette «fenêtre » devant laquelle se trouve un public concentré sur l'action théâtrale.

« Le surplus est inutile et peut être considéré comme la source des maux »

Claude Nicolas Ledoux

12 Le projet de Ledoux qui semblait relever de l'utopie appelait également la technique et les matériaux ductiles du $\mathrm{XX}^{\mathrm{ème}}$ siècle.

Ce qui paraissait hier chimérique entre aujourd'hui dans le domaine du possible.

\section{L'Avenir}

Après l'incendie du Théâtre de Claude Nicolas Ledoux le 29 avril 1958, Hélène Leclerc a écrit dans la revue « Histoire du Théâtre » un article qui n'a pas perdu de son actualité. "Après tant d'œuvres, non moins considérables, de Claude Nicolas Ledoux, le Théâtre de Besançon connaît à son tour la destruction quasi-totale. Nous ne reprendrons pas ici le thème qui court de "l'architecte maudit», la pérennité de Ledoux dépasse l'enveloppe périssable de son théâtre, car le souffle de ce génie précurseur rejoint, en les inspirant encore, les créations les plus audacieuses de notre temps. (...) Une reconstitution fidèle de l'œuvre de Ledoux ne serait-elle possible? La France ne pourrait-elle suivre l'exemple de l'Italie qui, après un désastre semblable a merveilleusement rénové l'une de ses plus belles salles, la Scala de Milan?» 
14 Partout dans le monde on connaît le Théâtre de Ledoux, on ne sait pas qu'il est à Besançon. Reconstruire ce théâtre, c'est au monde entier dire où il est, dire qu'il n'est pas que dans les livres...

15 Mais peut-être est-ce plus encore? C'est affirmer une volonté, celle d'associer comme Ledoux Théâtre et Démocratie qui, chacun sait, n'est pas que dans les livres.

\section{NOTES}

1. A l'occasion de la création de L'architecte de Jean Verdun par le C.D.N. de Franche-Comté, Coulisses $n^{\circ} 9$ a publié un dossier avec des articles sur le spectacle, l'auteur, et une biographie de C. Nicolas Ledoux. 